\title{
e-Phaïstos
}

e-Phaïstos

Revue d'histoire des techniques / Journal of the history

of technology

VIII-1 | 2020

Écomusée : une expansion internationale

\section{Quelle politique muséale pour la promotion de la diversité des expressions culturelles au Sénégal?}

What Museum Policy to Promote Cultural Diversity in Senegal?

\section{Boubacar Thioye}

\section{(2) OpenEdition}

Journals

Édition électronique

URL : http://journals.openedition.org/ephaistos/7627

DOI : 10.4000/ephaistos.7627

ISSN : 2552-0741

Éditeur

IHMC - Institut d'histoire moderne et contemporaine (UMR 8066)

\section{Référence électronique}

Boubacar Thioye, «Quelle politique muséale pour la promotion de la diversité des expressions culturelles au Sénégal ? », e-Phaïstos [En ligne], VIII-1 | 2020, mis en ligne le 29 avril 2020, consulté le 06 mars 2021. URL : http://journals.openedition.org/ephaistos/7627 ; DOI : https://doi.org/10.4000/ ephaistos.7627

Ce document a été généré automatiquement le 6 mars 2021.

Tous droits réservés 


\title{
Quelle politique muséale pour la promotion de la diversité des expressions culturelles au Sénégal ?
}

What Museum Policy to Promote Cultural Diversity in Senegal ?

\author{
Boubacar Thioye
}

«Au moment où l'Afrique a récupéré son

indépendance, au moment où l'Afrique se cherche, les dirigeants africains devraient se pencher sur le problème de l'utilisation des traditions s'ils veulent créer ou recréer une

Afrique africaine ", Amadou Hampathe Ba

1 Le $7^{e}$ Workshop international organisé par le Master TPTI (Techniques, Patrimoine, Territoires de l'industrie) pose une fois de plus la question de la valorisation du ou des patrimoines. Poser la thématique quel patrimoine pour quelle valorisation, c'est reconnaître le caractère pluridisciplinaire du patrimoine, des différentes formes qu'il peut revêtir, mais surtout de sa nécessaire valorisation. Défini comme l'ensemble de ce qu'une société entend conserver des traces de son passé dans le dessein de le transmettre aux générations futures, le patrimoine est à la fois matériel et immatériel. Définition vague car rien ne dit à quoi se limite ce patrimoine. Qu'est-ce qui fait patrimoine? Comment transmettre ce patrimoine? Autant de questionnements qui se posent en vue d'une réflexion dans un contexte de mondialisation et de recomposition des sociétés.

2 Notre axe de recherche se positionne à partir des différentes méthodes mises en œuvre pour la conservation et la transmission du patrimoine. Notre préoccupation centrale dans ce questionnement résulte $d u$ fait que, dans le contexte de mondialisation, les rapports de domination se ressentent jusque dans les manières de conserver le patrimoine dans des sociétés différentes. Loin de nous l'idée de critiquer mais de faire comprendre que la réalité de la culture impose à chaque société ses méthodes de 
valorisation, parce que les codes de communication ethniques sont différents d'une société à une autre.

3 Si le modèle du musée a fini de démontrer ses preuves en matière de gestion et de valorisation du patrimoine en Europe, force est de reconnaitre qu'il rencontre toutes les difficultés pour s'imposer en Afrique. Si l'ancien président du Mali, alors qu'il était ministre de la culture, s'exprimait en ces termes : « Il faut tuer le modèle occidental du musée en Afrique pour que s'épanouissent de nouveaux modèles de conservation et de promotion du patrimoine ", c'est que la reproduction à l'identique du musée n'est pas adapté à la culture africaine. C'est dans cette mouvance que nous envisageons de travailler sur les politiques muséales au Sénégal pour la promotion de la diversité des expressions culturelles. Confronté à une grande diversité ethnique et religieuse, le Sénégal, au lendemain de son accession à l'indépendance, a initié des politiques culturelles afin de cimenter l'identité nationale. Quelle place a occupé la question muséale dans ces différentes politiques culturelles? Quel impact a-t-elle eu sur les populations concernées? Quel bilan peut-on en tirer?

\section{Un aperçu de la diversité culturelle sénégalaise}

4 «Le Sénégal est un pays de l'Afrique occidentale, membre de la Communauté économique des États de l'Afrique de l'Ouest (CEDEAO) et de l'Union économique et monétaire des États d'Afrique de l'Ouest (UEMOA). Le Sénégal est une République laïque, démocratique et sociale, qui assure l'égalité de tous les citoyens devant la loi, sans distinction d'origine, de race, de sexe, de religion et qui respecte toutes les croyances. Sur le plan de l'organisation territoriale, il est composé actuellement de 14 régions, 45 départements, 172 communes et 384 communautés rurales. Ces collectivités locales, personnes morales de droit public, sont administrées librement par des citoyens élus au suffrage universel. La population sénégalaise est estimée à 128551531 habitants en 2011, avec un taux d'accroissement intercensitaire de 2,5\%. Cette population est essentiellement jeune (55\%) avec un âge médian est de 19 ans. Elle est composée d'une dizaine d'ethnies inégalement répartie dans l'espace.» (Enquête Myworld au Sénégal $2015: 3$ ).

5 Le 7 novembre 2006, en ratifiant la convention de 2005 sur la protection et la promotion de la diversité des expressions culturelles, le Sénégal a réaffirmé sa politique de promotion des libertés d'expressions, gage d'une démocratie vivante. L'histoire de la diversité culturelle au Sénégal est étroitement liée à celle du peuplement même du pays. « Nous sommes en réalité un pays de passages et de rencontres, de métissages et d'échanges » disait Léopold Sédar Senghor.

6 Il nous faut comprendre la notion d'ethnie avant de présenter celles qui constituent le paysage culturel sénégalais. Nous utiliserons la définition de Bromlei qu'en donne Matar Diouf dans son ouvrage Le Sénégal, les ethnies, la nation, qui affirme : "L'ethnie est un ensemble stable d'êtres humains, constitué historiquement sur un territoire déterminé, possédant des particularités linguistiques, culturelles (et psychiques communes et relativement stables), ainsi que la conscience de leur unité et de leur différence des autres formations semblables (conscience de soi) fixée dans l'autoappellation (ethnonyme) » (Diouf $1994: 10$ ). L'auteur note aussi que la langue et la culture peuvent être les conditions nécessaires de détermination de l'ethnie. S'y ajoutent les conditions suffisantes comme l'endoperception (comment les membres de 
l'ethnie se perçoivent) et l'exoperception (comment les membres de l'ethnie sont perçus par les autres). «L'ethnicité sera l'autoreprésentation, l'auto-identification du groupe, une quête d'identité collective, au plan culturel, et parfois au plan politique. » (Ibidem)

\section{Le paysage culturel sénégalais}

7 Le paysage culturel du Sénégal compte cinq grands groupes ethniques avec des sousgroupes et des ethnies autochtones (Bassari, Bedik). À ces groupes, il faut ajouter les nouveaux groupes issus des migrations et de la concurrence économique des pays développés dans les pays du tiers monde. Ce sont des immigrés (Chinois, Libanais, Français, Américains...) mais aussi des ethnies de la sous-région (Maliens, Guinéens, Mauritanien, Ivoiriens...).

\section{Les Wolofs}

8 Issus du royaume Djolof fondé au XVI ${ }^{\mathrm{e}}$ siècle, les Wolofs se dispersèrent vers le XVI ${ }^{\mathrm{e}}$ siècle. Ils constituent l'ethnie dominante et sont en majeure partie islamisés. Leur système de castes est toujours présent, mais il est lié à une spécialisation professionnelle. Ainsi il est possible de situer le métier d'une personne en se basant sur son nom patronymique. Un "Thiam " est à $90 \%$ bijoutier alors qu'un "Seck» est la plupart du temps griot ou homme de la parole. Les Wolofs sont agriculteurs (arachide), bijoutiers, cordonnier. Ils sont nombreux dans l'administration. Dans le groupe des wolofs, on retrouve le sous-groupe des Lébous, concentrés dans la région du Cap-Vert, dont la caractéristique principale reste la relation mythique avec la mer, ainsi qu'avec la médecine mystique. Ils sont appelés les peuples de l'eau.

\section{Les Alpoulhar}

9 Le groupe des Alpoulhar est composé de deux ethnies: les Peuls et les Toucouleurs. Leur langue est le poulaar. Les Peuls sont des bergers nomades. Ils se sont répandus à travers toute l'Afrique de l'Ouest et portent plusieurs noms : Foula, Foulani, Foulbé ou Poulo, etc. On les retrouve au Mali, au Burkina Faso, en Gambie, en Namibie, en Éthiopie, au Niger, au Nigeria. Leur dispersion met en cause les frontières en Afrique imposées par la colonisation. Les Toucouleurs se retrouvent sur la rive gauche de la vallée du fleuve Sénégal (Podor, Matam, Bakel...). Peuple de bergers, ils furent les premiers à embrasser la religion musulmane. On retrouve dans ce groupe les Laobés qui sont spécialisés dans la sculpture sur bois.

\section{Les Serer (Sérères)}

10 Deuxième ethnie du Sénégal après les Wolofs, les Sérères vivent de la culture du mil et de l'arachide ainsi que de la pêche. Leur mode de vie reste très marqué par l'animisme, quoiqu'ils aient pour la plupart adopté la religion chrétienne. Ils se concentrent principalement dans la région du Sine et du Saloum et sur la Petite-Côte. Le président Senghor est un descendant de cette tribu. Le groupe sérer se compose de deux principales entités de Serer, dites Serer Cangin (Noon, Ndut, Safi, Paloor) et Serer Siin. Le Cangin est dispersé dans la région de Thiès, tandis que le Siin est majoritaire dans la région de Fatick. 


\section{Les Diolas}

11 Ethnie de la Basse-Casamance, les Diolas vivent dans des régions forestières où ils cultivent le riz, qui sert de base à leur alimentation. Ce sont également les spécialistes de la culture des palmiers et de la récolte du vin de palme. Ils sont essentiellement animistes. Le groupe Diola est constitué de plusieurs variétés linguistiques (fogny, erring, bayot et flup).

Fig. 1. Le travail au kadiandou dans les rizières

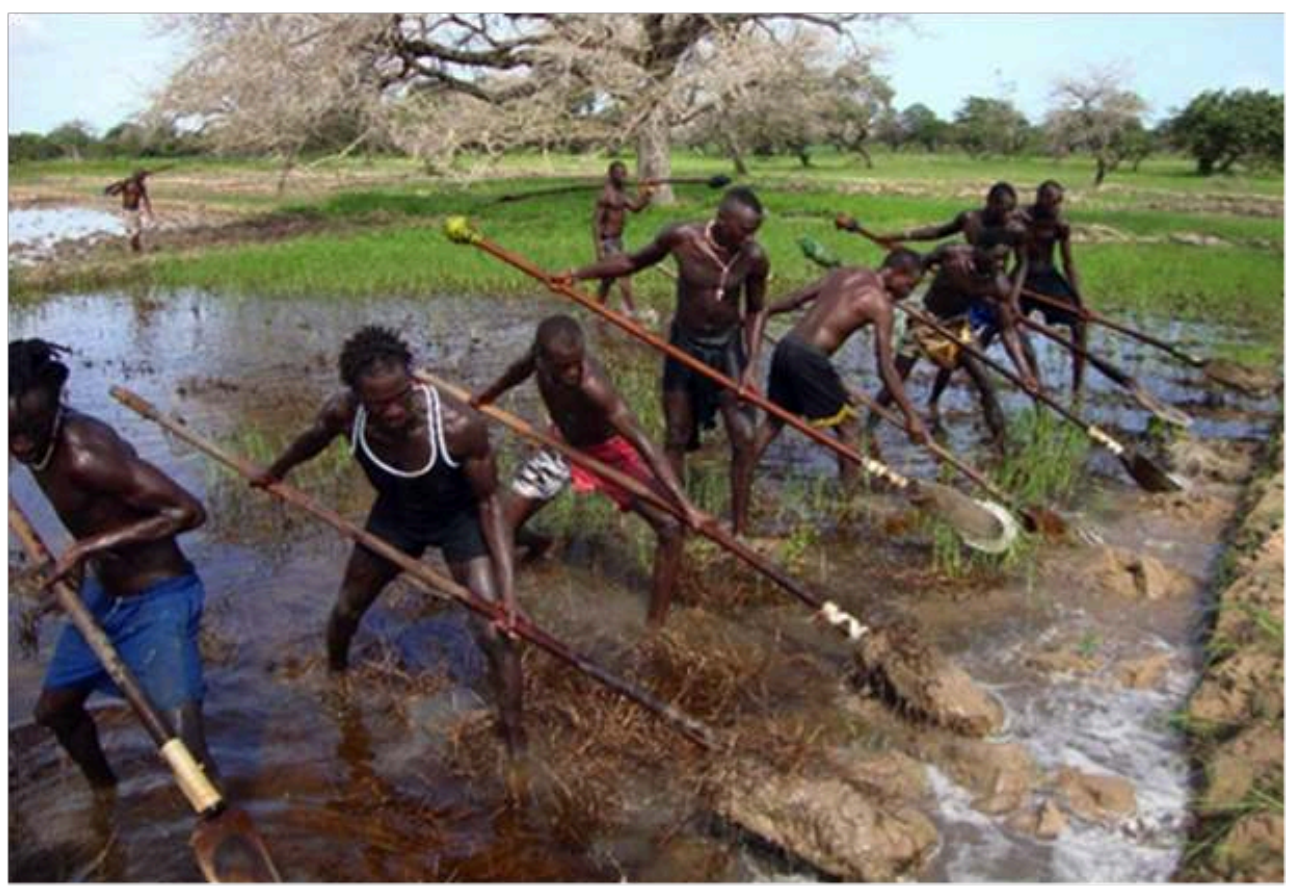

Document fourni par l'auteur

\section{Le groupe mandé}

12 Le groupe mandé comprend les Mandinkas, les Soninkes et les Bambaras, auxquels s'ajoutent les Manlinkes et Jaaxantes qui, bien que considérés comme des groupes ethniques différents, communiquent entre eux facilement. Ils occupent la région du Sénégal oriental.

\section{Le pays Bassari}

«Outre ces cinq grands groupes, d'autres groupes autochtones -dits minoritaires- sont présents sur le territoire sénégalais. Il s'agit des Maures dans les régions du nord, des Manjaks, des Balants, des Mankaañs et des Baynuks en Casamance, des Bassaris, des Koniagis, des Bediks et des Bajaraykes dans le Sénégal oriental. Notons aussi la présence du créole, du portugais de Casamance et de l'Arabe » (Cissé 2011).

Parmi ses minorités ethniques, les Bassaris ont été classés en 2012 patrimoine mondial de l'Unesco. "Situé dans le sud-est du Sénégal, le pays Bassari comprend trois régions géographiques différentes : celle des Bassaris, zone de Salémata, celle des Bédiks, zone de Bandafassi et celle des Peuls, zone de Dindéfello, présentant chacune des traits 
morphologiques particuliers. Les peuples Bassari, Peul et Bédik se sont installés entre le $\mathrm{XI}^{\mathrm{e}}$ et le $\mathrm{XIX}^{\mathrm{e}}$ siècle. Ils ont développé des cultures spécifiques, en symbiose avec l'environnement naturel. Le paysage bassari est organisé en terrasses et en rizières, entrecoupées de villages et de hameaux. Les villages des Bédiks sont formés de groupes denses de huttes aux toits de chaume pentus. Les expressions culturelles de ses habitants manifestent des traits originaux dans leurs pratiques agropastorales, sociales, rituelles et spirituelles et représentent une réponse exceptionnelle, originale aux contraintes imposées par l'environnement et aux pressions anthropiques. Le site présente un paysage multiculturel extrêmement bien conservé, abritant des cultures autochtones originales et toujours vivantes ». (UNESCO 2012)

\section{Discours interethnique}

15 «Sur la vingtaine de langues parlées au Sénégal, six seulement ont été promues au statut de langues nationales. Ce sont : le wolof, le serer, le pulaar, le joola, le mandinka et le soninké et cela en vertu du décret 68-871 du 24 juillet 1968 relatif à la transcription des langues nationales, revu par le décret du 21 mai 1971 et amendé par le texte de 1985 relatif au découpage des mots et autres règlements orthographiques. Ces langues sont toutes dotées d'un système de transcription (alphabet officiel en caractères latins). Mais seul le français a le statut de langue officielle. » (Cissé 2011)

16 Le fait marquant de la pluralité ethnique au Sénégal réside dans les rapports interethniques qui existent entre elles. Nous entendons donc par relations (interethniques), les rapports entre les peuples ou ethnies dont la société nationale sénégalaise est formée.

17 Dans la vie sociale du Sénégal comme de tout autre pays, on peut distinguer une vie historique, pour ainsi dire, officielle et une vie quotidienne. Dans la vie officielle, les ethnies en tant que telles ne sont pas prises en considération : les ministres, les fonctionnaires, les employés sont nommés et affectés sans qu'on tienne compte de leurs origines ethniques, excepté le théâtre national Daniel Sorano où la prise en compte des origines a toute son importance. En revanche, dans la vie quotidienne, cette origine joue un grand rôle. Dans les conversations courantes, on interpelle souvent l'interlocuteur en tant que Toucouleur, Diola, Serer, Peul ou Wolof. Pour qualifier la personnalité d'un homme ou d'une femme, on se réfère fréquemment à son appartenance ethnique. Aussi une grande part des plaisanteries si nombreuses dans les entretiens des Sénégalais portent sur des qualités et des défauts imputés à chacun en raison de son ethnie d'origine. Il n'y a entre les ethnies sénégalaises ni conflits ni tensions. Mais il existe chez chaque Sénégalais un ensemble de stéréotypes qui constituent comme un portrait-robot de sa propre ethnie et des portraits robots des autres ethnies.

18 Le fait marquant, dirait-on, ou le charme de la cohésion sociale au Sénégal réside dans le système des parentés à plaisanterie entre ethnies. Notion inventée par Marcel Mauss, la parenté à plaisanterie consiste à faire semblant de créer un conflit avec le représentant d'une ethnie alliée. Ce pacte permet aux protagonistes de s'insulter, de se railler et de se bousculer à l'envie, sans risque de dérapage. "Au bureau, dans la rue, dans les transports en commun, en famille ou avec des amis, la parenté à plaisanterie anime la vie quotidienne. Cette pratique dépasse les clivages sociaux et donne lieu parfois à des situations incongrues $»^{1}$. Il n'est pas rare de voir dans une entreprise un 
jardinier ou vigile serer interpeller ironiquement son patron alpoular en lui rappelant qu'il est son esclave et vice-versa.

\section{Les politiques muséales sénégalaises}

19 L'histoire des politiques d'institutionnalisation de la culture au Sénégal n'est pas liée à l'accession à l'indépendance comme on a tendance à le présenter dans les écrits. Le premier musée a vu le jour à Saint Louis, capitale de l'Afrique Occidentale française, en 1863, avec le Gouverneur Faidherbe. Il était considéré comme un centre de recherche sur les cultures noires et sur l'histoire africaine, mais aussi un espace de mise en scène de la puissance française sur les colonies. L'idée de la théorie de la table rase et de l'exploitation apparait bien dans le discours du gouverneur Faidherbe cité par Anne Gaugue : «Ce musée industriel, ethnologique et d'histoire naturelle doit répondre une double préoccupation d'éducation de la population et de mise en valeur de la colonie. Présentant les produits si nombreux et si variés que le Sénégal possède dans les différents règnes de la nature, ce musée doit non seulement aider puissamment à l'instruction de la population mais aussi conduire en certains cas à des découvertes précieuses pour le commerce, les arts et l'industrie » (Gaugue 1999 :728)

Les collections de ce musée sont transférées à Dakar jusqu'en 1936, avec la création de l'Institut Fondamental d'Afrique Noire (IFAN), où le projet de création d'un musée à proprement parler voit le jour. Deux ans plus tard, en 1938, est créé à Dakar le musée ethnographique de l'Afrique Occidentale. Les missions ne changent pas. C'est toujours dans la logique d'une mise en scène de l'ethnographie coloniale que s'inscrit ce musée fréquenté par les étrangers et les chercheurs coloniaux. Concrètement, le musée existait au Sénégal avant l'indépendance. Mais cette institution mise en place par le colonisateur était en rupture avec la population. Elle avait son public, ses codes de communications, ses normes, et n'avait rien de commun avec les pratiques sociales et culturelles des habitants.

$21 \mathrm{Au}$ lendemain des indépendances, une nouvelle forme de représentation de la culture émerge avec le Président Senghor. Convaincu que seule la décolonisation qui prenait en compte la dimension culturelle avant toutes les autres était celle qui valait, Senghor conduit, à partir de 1960, des politiques culturelles diverses pour redonner à l'homme noir sa dignité. On peut citer entre autres la création de l'École de Dakar sur les arts plastiques, la création du Musée Dynamique, le Théâtre National Daniel Sorano qui comporte trois ensembles (l'ensemble lyrique, le ballet national la Linguère et la compagnie dramatique), la Manufacture Sénégalaise des Arts Décoratifs qui a pour mission de s'inspirer du patrimoine immatériel pour le tissage des tapis. Des manifestations comme le 1er Festival Mondial des Arts Nègres en 1966, des expositions internationales avec des artistes comme Picasso, sont organisées à Dakar. Le commissariat aux expositions est créé pour la circulation des œuvres sénégalaises à l'international. Des mesures législatives, comme la loi du $1 \%$ relative à la décoration des bâtiments publics, font également partie de cette politique. L'analyse qu'on peut tirer de cette politique est qu'elle est fondée sur une idéologie longtemps défendue par l'ancien président qui s'est toujours battu pour le mouvement de la négritude. Ce mouvement qui a vu le jour à Paris avec Aimé Césaire, est défini comme l'ensemble des valeurs de civilisation du monde noir. On note des réussites dans certains domaines. C'est le cas avec la Compagnie du Théâtre National Daniel Sorano. Elle regroupe la 
presque totalité des ethnies du Sénégal qui travaillent dans la symbiose et l'interculturalité afin de représenter la sociabilité dans leur production artistique. Il n'en est pas de même dans les musées.

En 1980, le coût des ajustements structurels conduit Abdou Diouf, le successeur de Senghor à réduire le budget de la culture en faveur d'autres domaines comme l'économie, la santé et l'éducation. Néanmoins, il a été à l'origine de la création de la biennale internationale des arts de Dakar et des convergences culturelles qui donneront le Festival National des Arts et Cultures.

En 2000, la politique culturelle prend une nouvelle forme avec le président Abdoulaye Wade. Elle prend appui sur la construction d'infrastructures. La nouvelle donne avec la politique culturelle sous le régime de l'alternance, c'est que la base idéologique change de paradigme pour épouser celui de la renaissance africaine. On assiste à la construction de la place du millénaire, de la place du souvenir africain, du monument de la renaissance africaine, du projet du mémorial de Gorée...

Depuis 2012, la politique culturelle initiée par l'actuel président Macky Sall se fonde sur la territorialisation de la culture. C'est ainsi que des projets comme la promotion de la diversité des expressions culturelles, la création de nouvelles institutions culturelles comme les écomusées ou les villages communautaire ont été initiés. Dans ces différentes politiques, la question muséale est sans doute celle qui a eu le moins d'impact au niveau des populations concernées. Quelle analyse peut-on tirer de cette rupture?

\section{Un public dans les musées sénégalais?}

Le regard sur la question du public du musée est plus que problématique en Afrique et cela pour plusieurs raisons. C'est d'abord une conséquence de la nature du patrimoine qui est immatériel, car fondé sur une civilisation orale. Qui dit patrimoine immatériel se réfère donc plus à des systèmes de symboles. Compte tenu de ce caractère intangible, le musée, reçu en héritage de l'occident, ne pouvait à aucun moment transmettre la culture africaine quelles qu'aient été les tentatives. La transmission patrimoniale obéit à des normes, des codes, par conséquent à un capital symbolique et culturel. Les études sociologiques de Pierre Bourdieu ont démontré que le seul fait économique ne suffisait pas à définir le capital culturel. Il fallait prendre en compte les niveaux d'études, l'héritage culturel familial. Ce sont les habitus.

Malgré la présence de musées au Sénégal, le fait marquant est que cette institution est méconnue et non fréquentée par la population. Les multiples politiques culturelles initiées par les différents gouvernements respectifs n'ont pas réussi à atteindre ce public. Nous ne pouvons pas évoquer la question du volet économique car un coup d'œil sur l'actualité culturel du pays démontre que le citoyen sénégalais le plus pauvre est prêt à débourser 40 euros pour un spectacle de musique ou de théâtre alors que l'entrée au musée est fixée à 3 euros. Ainsi, la question que nous nous posons est la suivante : les musées sont-ils porteurs de la culture sénégalaise ? Une réponse positive s'impose de prime abord. L'approche à partir de laquelle cette culture est transmise estelle la bonne ? C'est là où se situe tout le problème. Le modèle occidental du musée demeure en déphasage avec les réalités africaines. Il faut imaginer un modèle apte à prendre en charge la dimension collective de la culture. Les cultures africaines sont communautaires. C'est pour cela que nous pensons que le modèle écomuséal paraît le 
seul qui puisse en rendre l'exactitude, tout en établissant le lien avec le public concerné.

\section{Le modèle écomuséal, outil de promotion de la diversité culturelle}

Soutenir la pertinence de la thèse du modèle écomuséal pour la promotion de la diversité des expressions culturelles, c'est d'abord expliquer le concept même d'écomusée.

Terme inventé par Georges Henri Rivière dans les années 1950, le modèle écomuséal est expérimenté en 1968 au Creusot-Montceau. Laboratoire, école, conservatoire, l'écomusée dépasse le concept classique de musée. On le définit comme "une institution culturelle, assurant d'une manière permanente, sur un territoire donné, avec la participation des populations, les fonctions de recherche, conservation, présentation et mise en valeur d'un ensemble de bien naturels et culturels représentatifs d'un milieu et des modes de vie qui s'y succèdent » (Ministère de la culture et de la communication, 1981).

Le concept est né dans un contexte marqué par le désir de renouveler en France le lien entre le musée et son public. Mais le plus important dans la définition est sans doute l'utilisation de la trilogie suivante: patrimoine, territoire et population). Dans l'élaboration des projets culturels au Sénégal, le processus « top down» « du haut vers le bas » a toujours primé sur le processus « bottom up ». L'approche écomuséale est, pour nous, la meilleure forme, car elle donne les pleins pouvoirs aux populations des régions et aux ethnies de décider de ce qui fait patrimoine. Elles sont avant tout les acteurs et les bénéficiaires. On est devant le principe même de la démocratisation culturelle qui a d'ailleurs connu des succès importants à travers le monde, en particulier en Amérique latine. Mais le continent africain a lui aussi porté à leur terme de nombreux projets propres à cette logique. On pense notamment à l'importance prise par le concept d'écomusée, en Afrique du Sud. À ce propos, les réalisations mises en avant dans le cadre du Aha Lesedi Cultural Village constitue un point d'appui sur lequel une réflexion proche pourrait être menée dans le cadre du Sénégal. Cette réalisation sud-africaine confronte les modes de vie de cinq des ethnies les plus caractéristiques du pays, mais avec sans doute une approche touristique trop exacerbée.

Dans le cas du Sénégal, des espaces publics en passant par les paysages culturels façonnés par les activités culturelles jusqu'à la poésie orale, l'application du modèle de l'écomusée doit favoriser la mise en valeur des savoirs, des savoirs faire techniques et culturels propres aux différentes ethnies du pays. Ainsi, des écomusées dans les régions du Sénégal permettraient une collecte non négligeable sur les techniques traditionnelles selon les domaines et pour chaque ethnie (agriculture, riziculture, forge, élevage, pèche, architecture, tissage, vannerie, sculpture, cordonnerie, bijouterie...), mais aussi sur l'imaginaire collectif des ethnies. 


\section{BIBLIOGRAPHIE}

AZIZA Mohamed, Patrimoine culturel et création contemporaine, Dakar, Nouvelles Éditions Africaines, 1978

BABA Kake Ibrahima, « Culture africaine, identité Culturelle, développement, dialogue des cultures », Éthiopiques, n40-41, 1985

BONNOT Thierry, La vie des objets, Paris, Éditions de la Maison des Sciences de l'Homme et Mission du Patrimoine Ethnologique, 2002

CHEVALLIER Denis, Savoir-faire et pouvoir transmettre, Paris, Éditions de la Maison des sciences de l'Homme, 1996

CISSÉ Mamadou, «Langues et glottopolitique au Sénégal », Ethiopiques n87, 2011, en ligne : http://ethiopiques.refer.sn

DIAGNE Mamoussé, Critique de la raison orale. Les pratiques discursives en Afrique, Paris, Éditions Karthala, 2005

DIOUF Makhtar, Le Sénégal, les ethnies, la nation, Paris, L'Harmattan, 1994

Enquête Myworld 2015 au Sénégal. Les priorités de développement des sénégalais, Ministère du Plan, Direction général du Plan, Septembre 2013, En ligne, https://www.undp.org/content/dam/ senegal/docs/OMD/undp-sn-Myworld\%20Senegal\%202015_Rapport\%20final.pdf

GARÇON Anne-Françoise, L'imaginaire et la pensée technique. Une approche historique, $\mathrm{XVI}^{e}-\mathrm{XX}^{e}$ siècle, Paris, Classiques Garnier, 2012

GARÇON Anne-Françoise, CARDOSO DE MATOS Ana, FONTANA Giovanni Luigi (dir.), L'aluminium et la calebasse, Belfort, Éditions de l'UTBM, 2013

GAUGUE Anne, « Musées et colonisation en Afrique tropicale (Museums and Colonization in Tropical Africa ), Cahiers d'études africaines, vol.39, n¹55-156, 1999, p.727-745

GAUGUE Anne, «Exposer l'histoire nationale : des musées forcément subjectifs », Africultures, vol. $1, n^{\circ} 70$, p. 65-70, 2007, DOI : 10.3917/afcul.070.0065

HUCHARD Ousmane Sow, La culture, ses objets témoins et l'action muséologique. (Sémiotique et témoignage d'un objet témoin : le masque Kanaga des Dogons de Sanga), Dakar, Éditions le Nègre International, 2010

KANTE Nambala, Forgerons d'Afrique Noire. Transmission des savoirs traditionnels en Pays Malinké, Paris, L'Harmattan, 1993

LERICH Frédéric, DAVIET Sylvie, SIBERTIN Mariette, ZULIANI Jean-Marc (dir.), L'économie culturelle et ses territoires, Toulouse, Presses Universitaires du Mirail 2008

Les recherches en sciences humaines et sociales pour les développements culturels, Communications au séminaire de concertation des responsables des instituts nationaux de recherche en sciences humaines et sociales organisé par l'I.C.A (Institut Culturel Africain) du 6 au 10 Décembre 1976 à Dakar

MINISTÈRE DE LA CULTURE ET DE LA COMMUNICATION, Charte des écomusées. Instructions du 4 mars 1981, 1981 
THIOYE Boubacar Obeye, Processus de transmission des savoirs agricoles chez les Sereer au Sénégal : projet de création d'un écomusée du terroir, Master Thesis, Master Erasmus Mundus TPTI, Université d'Èvora, 2015, http://hdl.handle.net/10174/18714

UNESCO, « Pays Bassari : paysages culturels Bassari, Peul et Bédik », Liste du Patrimoine mondial. Paysage culturel, 2012, en ligne : https://whc.unesco.org/fr/list/1407

VARINE Hugues de, Les Racines du Futur. Le patrimoine au service du développement local, Châlonssur-Saône, Asdic, 2002

\section{NOTES}

1. "Le bienfait des railleries ethniques", Le nouvel Afrik.com, 5 mars 2004, https:// www.afrik.com/le-bienfait-des-railleries-ethniques.

\section{RÉSUMÉS}

La réalité de la culture impose à chaque société ses méthodes d'évaluation. En effet, les codes ethniques de communication sont différents d'une société à l'autre. Le musée est devenu un outil majeur de gestion et de valorisation du patrimoine en Europe. Mais l'institution muséale est difficile à établir en Afrique. Or, dans le contexte de la mondialisation, les relations de domination se ressentent même dans la manière de préserver le patrimoine dans différentes sociétés. Il est donc intéressant d'étudier les politiques muséales développées au Sénégal pour promouvoir la diversité des expressions culturelles. Face à une grande diversité ethnique et religieuse, le Sénégal, au lendemain de son indépendance, a initié des politiques culturelles pour cimenter l'identité nationale. Quelle place la question muséale a-t-elle occupée dans ces différentes politiques culturelles? Quel a été son impact sur les populations concernées? L'écomusée est-il un bon outil pour la valorisation des cultures africaines ? C'est ce que nous essayons d'analyser dans cet article.

The reality of culture imposes on each society its methods of evaluation. Indeed, the ethnic codes of communication are different from one society to another. The museum has become a major tool for managing and promoting heritage in Europe. But the museum institution is difficult to establish in Africa. In the context of globalization, however, relations of domination are felt even in the way of preserving the heritage in different societies. It is therefore interesting to study the museum policies developed in Senegal to promote the diversity of cultural expressions. Faced with great ethnic and religious diversity, Senegal, in the aftermath of its independence, initiated cultural policies to cement the national identity. What place has the museum question occupied in these different cultural policies? What was its impact on the populations concerned? Is the eco-museum a good tool for promoting African cultures? This is what we are trying to analyze in this article. 
INDEX

Mots-clés : histoire des techniques, écomusée, identité culturelle, politique culturelle

Keywords : history of technology, eco-museum, cultural heritage, cultural identity

\section{AUTEUR}

\section{BOUBACAR THIOYE}

Boubacar Obeye Thioye est diplômé du Master Erasmus Mundus TPTI, promotion 7, « Madiba Mandela ", 2015. Son mémoire de master était consacré au projet de création d'un écomusée pour la mise en valeur et la sauvegarde des savoirs agricoles chez les Serer du Sénégal. 